trialkylboranes ; carbenes ; ethynylation; isomerization; carbon-carbon bond formation; and a few miscellaneous preparations including diazomethane and two peroxyacids; a few pages at the end are devoted to simple techniques such as distillation and adsorption chromatography.

This might seem to provide fairly wide coverage, with up to ten individual preparations under each heading, but there are a few serious omissions. The experiments chosen reveal a preoccupation with alicyclic chemistry; there are far fewer examples from acyclic chemistry, only a few involving aromatic chemistry and hardly any from heterocyclic chemistry; nor are there any involving amino acids. Several examples from these areas would have restored the balance which is lacking. A few more examples involving modern organometallic reagents are also called for. In a few instances the procedure recommended is neither the most successful nor the simplest available. For example, hydrolysis of the bromide on page 152 proceeds perfectly well in aqueous solution without adding silver nitrate; and the Wittig procedure on page 106 gives a poor yield of methylenecyclohexane. It seems not to be widely known that replacement of the solvent ether by diglyme in this experiment improves the yield to almost the theoretical value. Generally, however, the book fulfils its purpose, which is to provide an introduction to organic synthesis for beginning graduate students and advanced undergraduates.

M. A. MCKervey

\section{Day Length and Wobble}

Rotation of the Earth. Edited by Paul Melchoir and Shigeru Yumi. With the cooperation of Lady Jeffreys. (International Astronomical Union/Union Astronomique Internationale. Symposium No. 48, held in Morioka, Japan, May 1971.) Pp. xxii + 244. (D. Reidel : Dordrecht, 1973.) Dfl.40.

THIS book is based on papers given at the International Astronomical Symposium No. 48, on the "Rotation of the Earth", held in Morioka, Japan, in May 1971. Attending were geophysicists and astronomers from North America, Europe and Japan, as well as several scientists from the USSR. The book begins with a review by one of the editors, Dr P. Melchoir, outlining the developments in the field since the publication in 1960 of Walter Munk and Gordon Macdonald's classic by the same title.

Many of the papers were concerned with improvements in determining the
Earth's polar motion (wobble) and changes in the length of day ( $\triangle \mathrm{LOD}$ ) by optical observations of the "fixed stars". Several new sources of information on wobble and $\triangle \mathrm{LOD}$ of the next decade, however, show potential for high accuracy. These include observations of near-Earth geodetic satellites, lunar laser ranging experiments and the use of very long baseline interferometry. In this respect, this volume provides invaluable references to the literature relating to these new developments.

There are also several papers discussing how internal motions of the Earth, such as core-mantle interactions and continental drift, can be measured directly using these new techniques of fundamental astronomy. Of special note is B. D. Tapley and B. E. Schultz's rigorous stochastic treatment of changes in the Earth's rotation, leading directly to models compatible with radar tracking data from near-Earth satellites. The reader with a more speculative turn of mind will find satisfaction in learning of recent developments relating earthquakes, solar activity and observation of irregular changes in polar motion. Earthquakes are also discussed as a source of excitation to the well known, but poorly understood, 14-month Chandler wobble.

The proceedings of this conference, when combined with L. Mansinha's collection of papers from a 1969 NATO Advanced Study Institute on Earthquake Displacement Fields and the Rotation of the Earth (D. Reidel, Holland, 1970), make valuable references on progress since 1960 for workers in fundamental astronomy and geodynamics, and an important one for earth science, astronomical, and basic physics libraries.

\section{Stephen H. PlagemanN}

\section{Radiomythology}

Low-level Radiation. By Ernest J. Sternglass. Pp. xvi +166 . (Earth Island: London, February 1973.) $£ 2.50$.

IT must be presumed that Professor Sternglass never intended this book to be treated as a scientific work, otherwise he would not deliberately have misused scientific terms, used large numbers of very small units to give the impression of large quantities and been so highly selective in his use of the available data. Instead he has written a popular account, a story, of the ideas which lead him to conclude that the deleterious effects of low-level radiation from nuclear fall-out have been grossly under- estimated, particularly in respect to infant and foetal deaths. Moreover, he claims that he was positively obstructed by the US Atomic Energy Agency and several leading scientists in presenting these ideas at the Congressional hearings on the effects of nuclear fall-out and in the scientific press.

One reason for his hostile reception can be found in his claim that "the nature of modern science depends upon the free communication of even the most disturbing ideas, since only through their widest possible examination can the essential process of the gradual correction of errors be accomplished". If there were no more to it than this a few imaginative people could swiftly put an end to all scientific progress. In this book Professor Sternglass has himself produced enough disturbing ideas to employ a whole army of scientists but he has not produced sufficient evidence to show that such effort would be rewarding. It is not good enough merely for him to provide the ideas that certain specific increases in fall-out might have been the cause of certain specific adverse changes in the health of selected populations. We need to know whether or not these ideas stand up to criticism when all the available data are considered, and, for example, if there is a relationship between foetal death and such diverse insults as a brief exposure to ${ }^{131} \mathbf{I}$ and the slow release of ${ }^{90} \mathrm{Sr}$ from maternal bone, what is the unifying factor and how it can be quantified in relation to risk.

It is now a respectable hypothesis that all releases of radioactivity to the environment can be expected in the long term to increase the incidence of malignant disease and genetic mutations throughout the world. All that is at issue is the magnitude of those increases and this is a matter which is under constant review by such reputable bodies as the United Nations Scientific Committee on the Effects of Atomic Radiation. By presenting his claims now without a reasoned appraisal of the views of such bodies Professor Stern. glass can only further alienate the support of many scientists who honestly seek to find the true relationship between risk and low-level radiation. In this book he may have lost credibility even in the eyes of the most casual reader who, for example, will surely find it incredible that this man was actually waiting for the library doors to open in order to check that yet another trend in the US vital statistics supported his claims.

There is a real need for the relevant facts to be put in simple terms before the public. What a pity that Professor Sternglass, with his undoubted powers of popular expression, did not choose to present a more balanced view.

J. VenNART 